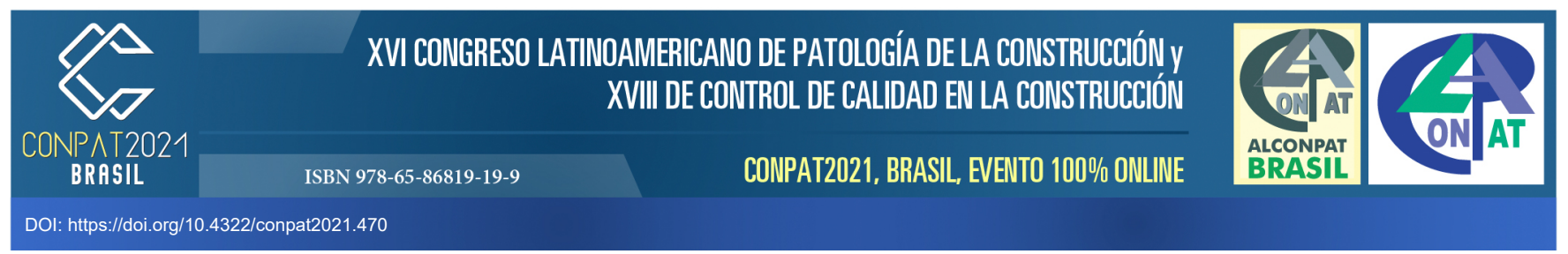

\title{
Manifestações Patológicas causadas por erros em concepção estrutural de edifícios altos: um estudo de caso
}

\author{
D.V.C. Teles ${ }^{1}$, D.M. Santos ${ }^{1}$, R.N. Cunha ${ }^{2 *}$, C.S. Vieira ${ }^{1}$, D.L.N.F. Amorim ${ }^{1,2}$ \\ *Autor para contato: rafael.cunha@ctec.ufal.br \\ ${ }^{1}$ Laboratório de Modelagem Matemática em Engenharia Civil, Universidade Federal de Sergipe, São Cristóvão, Brasil \\ ${ }^{2}$ Programa de Pós-Graduação em Engenharia Civil, Universidade Federal de Alagoas, Maceió, Brasil
}

\begin{abstract}
Resumo
A concepção é a etapa inicial de um projeto estrutural onde busca-se posicionar os mais diversos elementos de um sistema, tais como as vigas, os pilares, as lajes e entre outros. Escolhas inadequadas nessa etapa podem causar grandes problemas futuros para as edificações, como a perda da estabilidade global, que acarreta o aumento dos esforços internos, que pode levar ao surgimento de manifestações patológicas. Neste sentido, este trabalho buscou, por meio de uma análise estrutural, verificar a influência da concepção estrutural de um edifício brasileiro nas manifestações patológicas que vieram a surgir na etapa de pós-ocupação.
\end{abstract}

Palavras chave: Concreto armado; Manifestações patológicas; Edifícios altos; Estabilidade estrutural.

Teles, D. V. D., Santos, D. M., Cunha, R. N., Vieira, C. S., Amorim, D. L. N. F. 2021, Manifestações patológicas causadas por erros em concepção estrutural de edifícios altos: um estudo de caso. Proceedings of XVI Congreso Latinoamericano de Patología de la Construcción y VIII Congreso de Control de Calidad en la Construcción. v. X, pp. X-X. 


\begin{abstract}
The conception is the initial stage of a structural design, which it seeks to position the most diverse elements of a system, such as beams, columns, slabs and others. Inadequate choices at this stage may cause major future problems for buildings, such as the loss of global stability, which leads to an increase in internal forces, that may lead to the emergence of pathological manifestations. In this sense, this paper searched, through a structural analysis, to verify the influence of the structural design of a Brazilian building in the pathological manifestations that came to appear in the postoccupation stage.
\end{abstract}

Keywords: Reinforced concrete; Pathological manifestations; Tall buildings; Structural stability.

\title{
Resumen
}

La concepción es la etapa inicial de un diseño estructural, que busca posicionar los más diversos elementos de un sistema, como vigas, pilares, losas y otros. Las elecciones inadecuadas en esta etapa pueden generar grandes problemas futuros para los edificios, como la pérdida de la estabilidad global, que conduce a un aumento de las fuerzas internas, que pueden conducir a la aparición de manifestaciones patológicas. En este sentido, este trabajo buscó, con el uso de análisis estructural, verificar la influencia del diseño estructural de un edificio brasileño en las manifestaciones patológicas que llegaron a aparecer en la etapa de post-ocupación.

Palabras clave: Concreto reforzado; Manifestaciones patológicas; Edificios altos; Estabilidad estructural.

\section{INTRODUÇÃO}

No Brasil, os sistemas estruturais mais utilizados em edifícios de concreto armado de 15 a 20 pavimentos são formados somente por pórticos ou sistemas duais, ou seja, compostos por pórticos e pilares-parede ou um núcleo rígido. No primeiro caso, o sistema de contraventamento é composto apenas por pilares e vigas, onde por meio da rigidez à flexão do conjunto torna-se possível resistir a atuação das cargas laterais e verticais. Enquanto no modelo de pórticos com núcleo rígido, combate-se as ações adicionando a vigas e pilares um núcleo estrutural com alta rigidez, constituído por um ou mais pilares com seção transversal de dimensões muito superiores aos demais elementos. A escolha de um adequado sistema estrutural é de suma importância para a obtenção de uma estrutura que possua boas características de segurança e durabilidade. Erros de concepção podem levar à instabilidade global nas estruturas, que podem causar o surgimento de manifestações patológicas nas edificações durante a sua vida útil.

Em muitos casos, as manifestações patológicas estão associadas ao comportamento global da edificação, como recalques diferenciais das fundações e deslocabilidade lateral da construção. Métodos simplificados podem ser utilizados para avaliar a estabilidade global de estruturas. A NBR 6118 (2014) indica o uso dos parâmetros $\alpha$ e $\gamma_{z}$ para esta avaliação. O primeiro foi desenvolvido por Beck e König (1966), enquanto que o segundo, por Franco e Vasconcelos (1991).

Foram realizados estudos relacionando ambos os parâmetros em diferentes estruturas (Carmo et al., 1995; Campoó et al., 2005; Menezes et al., 2020). 
O coeficiente $\gamma_{\mathrm{z}}$ tem sido muito utilizado devido à sua fácil aplicação em programas comerciais de cálculo estrutural. Apesar de simplificado, o método possui boa capacidade de estimar os esforços de segunda ordem das estruturas (Feitosa; Alves, 2015; Bueno; Loriggio, 2016; Freitas et al., 2016). Ainda assim, devido à importância da análise de estabilidade global de estruturas, outros trabalhos têm sido realizados propondo critérios similares (Vieira et al., 2017; Elwanger, 2019; Cunha et al., 2020). Neste sentido, uma avaliação incorreta da estabilidade pode acarretar em diversas manifestações patológicas na estrutura ou até mesmo o colapso (Jesus et al., 2020).

Um caso real de manifestações patológicas associadas à instabilidade estrutural foi apresentado por Fusco (1996), em que tal correlação se confirma por meio de um cálculo manual. Em seguida, Jesus et al. (2020) mostram como o coeficiente $\gamma_{z}$ ajuda a explicar as manifestações patológicas deste caso. Na verdade, Jesus et al. (2020) não se atêm às manifestações patológicas em si, pois se verificou por meio do coeficiente $\gamma_{\mathrm{z}}$ e de análises P- $\Delta$ que o edifício entraria em colapso mesmo sem a ação horizontal do vento, o que explica, em linhas gerais, as manifestações patológicas severas por toda a edificação. É importante notar que a estrutura não entrou em colapso porque foi feito um trabalho de recuperação com chapas metálicas e tirantes, conforme explica Fusco (1996), de modo a garantir a estabilidade global da edificação.

Diante do exposto, este trabalho tem como objetivo apresentar o estudo de caso sobre a influência da concepção estrutural nas manifestações patológicas do edifício apresentado em Fusco (1996). Para isso, foram avaliados os esforços internos, obtidos via simulações numéricas utilizando o modelo de pórtico espacial combinado a metodologia de grelha equivalente. Algumas manifestações patológicas são analisadas separadamente, de modo que sua ocorrência seja justificada com base na teoria das estruturas e nas diretrizes da NBR 6118 (2014).

\section{ESTUDO DE CASO: PADRÃO DE FISSURAÇÃO}

O estudo de caso apresentado neste trabalho trata de quatro edifícios de concreto armado idênticos que apresentaram as mesmas manifestações patológicas, conforme relatado em Fusco (1996). Os edifícios foram construídos simultaneamente, possuindo 15 pavimentos acima do nível do baldrame, além da cobertura, e dimensões de aproximadamente $47 \mathrm{~m}$ de altura, $32 \mathrm{~m}$ de largura e $12 \mathrm{~m}$ de comprimento. Dados como nome do empreendimento, projeto arquitetônico, data do projeto, projeto de fundações, detalhamento do projeto estrutural e maiores detalhes construtivos não são informados em Fusco (1996). A planta de forma do pavimento tipo, adaptada do trabalho anteriormente citado, é apresentada na Figura 1.

Foram notadas fissuras iniciais (tipo 1) em todos os pavimentos dos quatro edifícios quando ainda estavam em fase de construção (Figura 2). Estas fissuras eram visíveis na face inferior das lajes, mas só causaram preocupação após o final da obra devido ao alto número de reclamações dos proprietários dos apartamentos. Após uma investigação, observou-se que as fissuras cortavam as lajes face a face. 


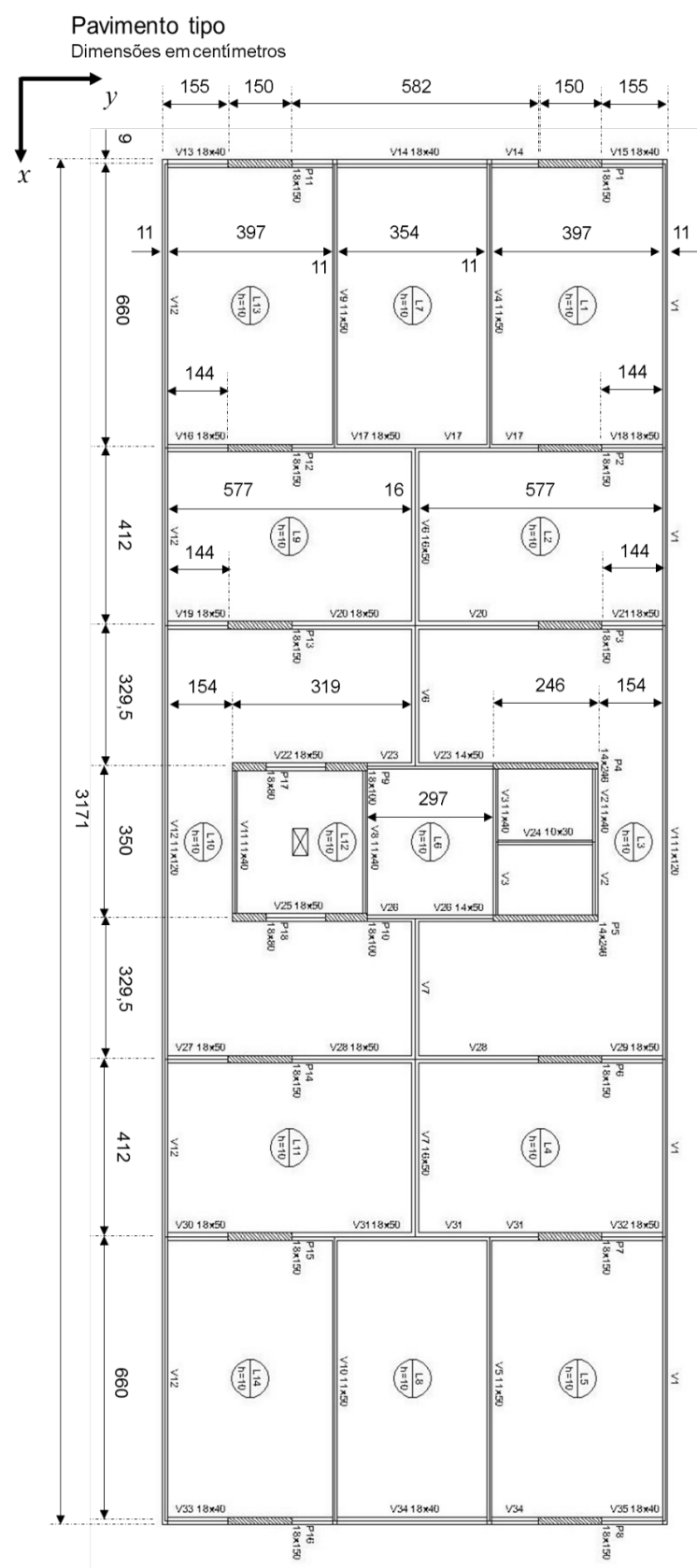

Figura 1. Planta de forma da estrutura. Fonte: adaptado de Fusco (1996).

Posteriormente, foi realizada uma vistoria inicial que constatou um nível bastante significativo de fissuração (Figura 3). Foram registradas fissuras verticais (tipo 2), rupturas localizadas em cruzamentos de alvenarias (fissuras tipo 3) e uma fissura em uma parede revestida (tipo 3'), além das fissuras de canto já relatadas anteriormente na Figura 2. Após esta vistoria, observações permanentes foram realizadas nos edifícios. 


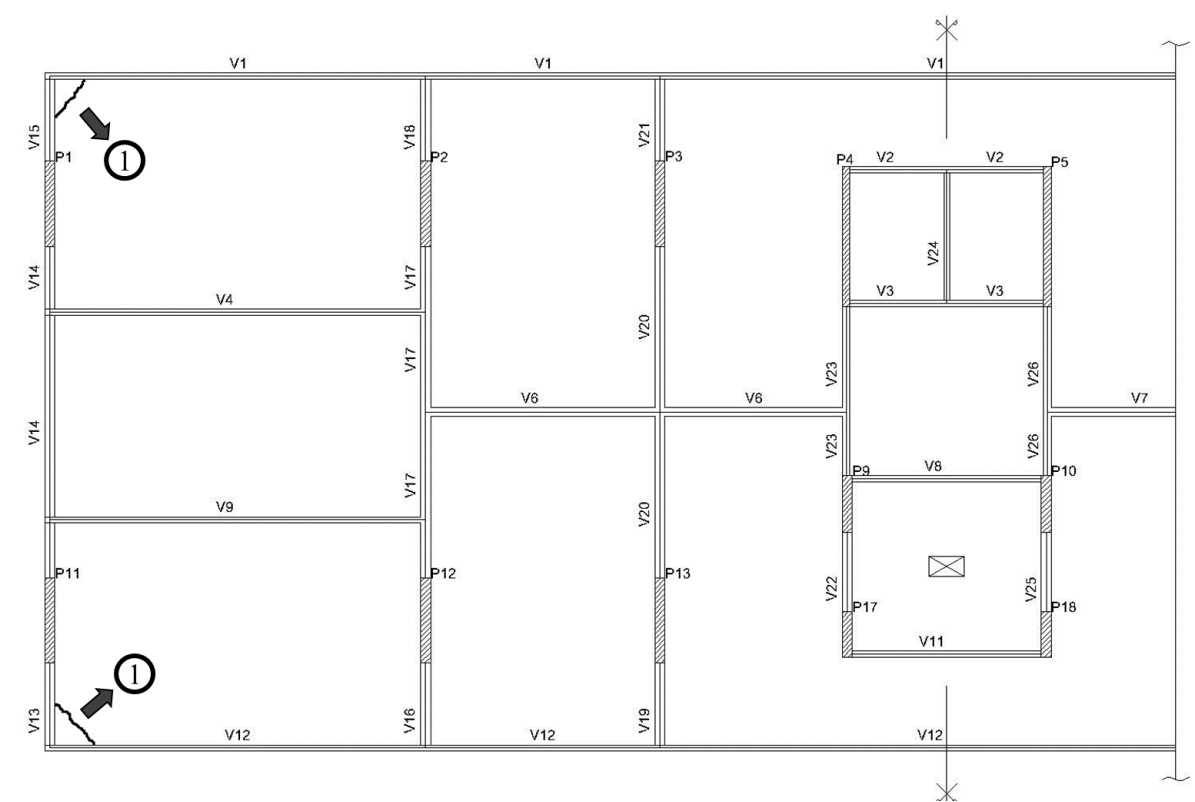

Figura 2. Observações iniciais relatadas. Fonte: adaptado de Fusco (1996).

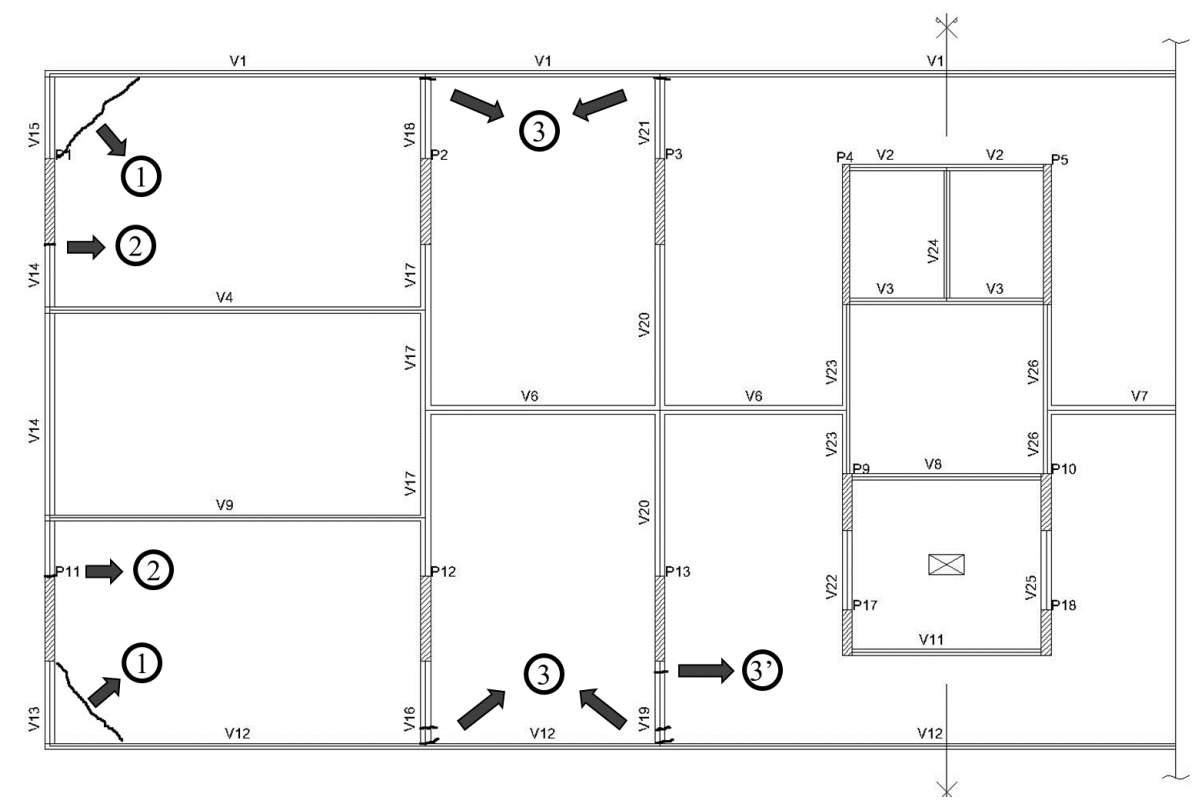

Figura 3. Dia 01: fissuras relatadas após vistoria. Fonte: adaptado de Fusco (1996).

Após oito dias de observação, a situação já tinha se agravado significativamente, ocorrendo a evolução das fissuras relatadas anteriormente e o surgimento de novas fissuras nos contatos entre alvenarias e peças estruturais (fissuras tipo 4 e 5). Surgiram também as primeiras rupturas das lajes situadas no eixo de simetria da estrutura (tipo 6) (Figura 4). 


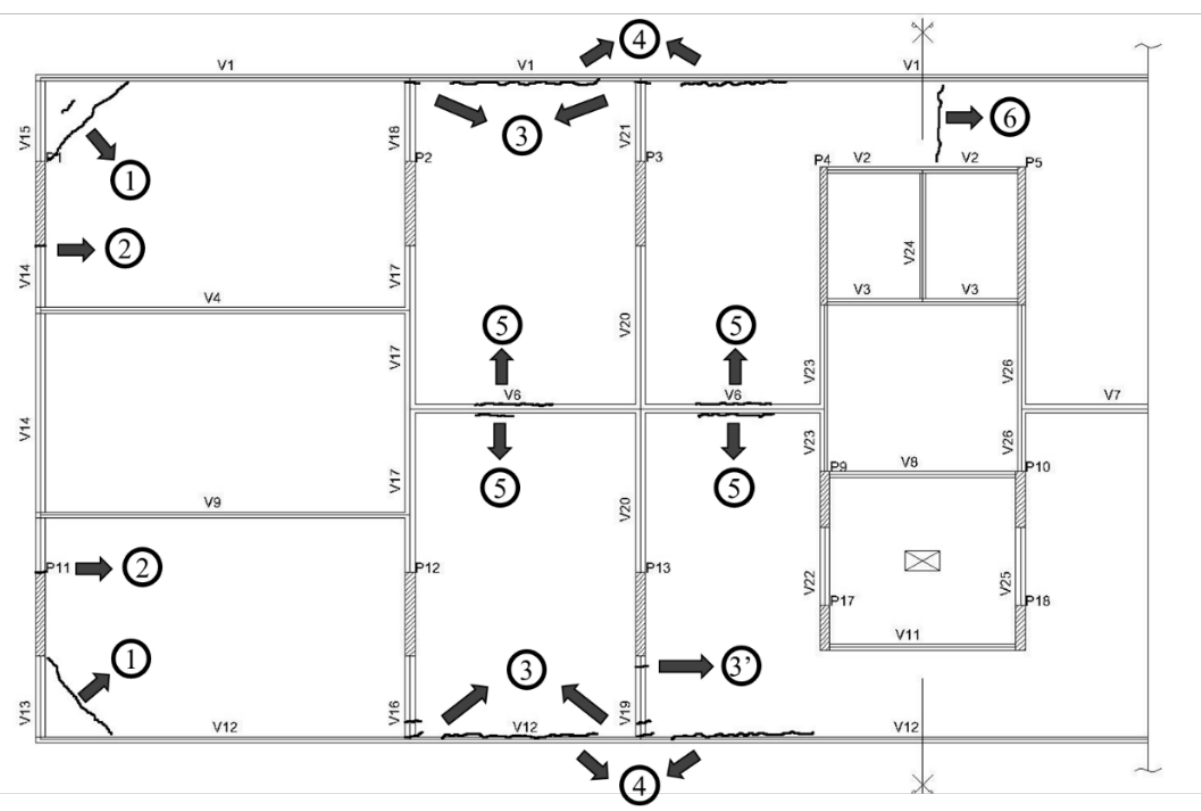

Figura 4. Dia 08: situação das fissuras relatadas e aparecimento de novas fissuras. Fonte: adaptado de Fusco (1996).

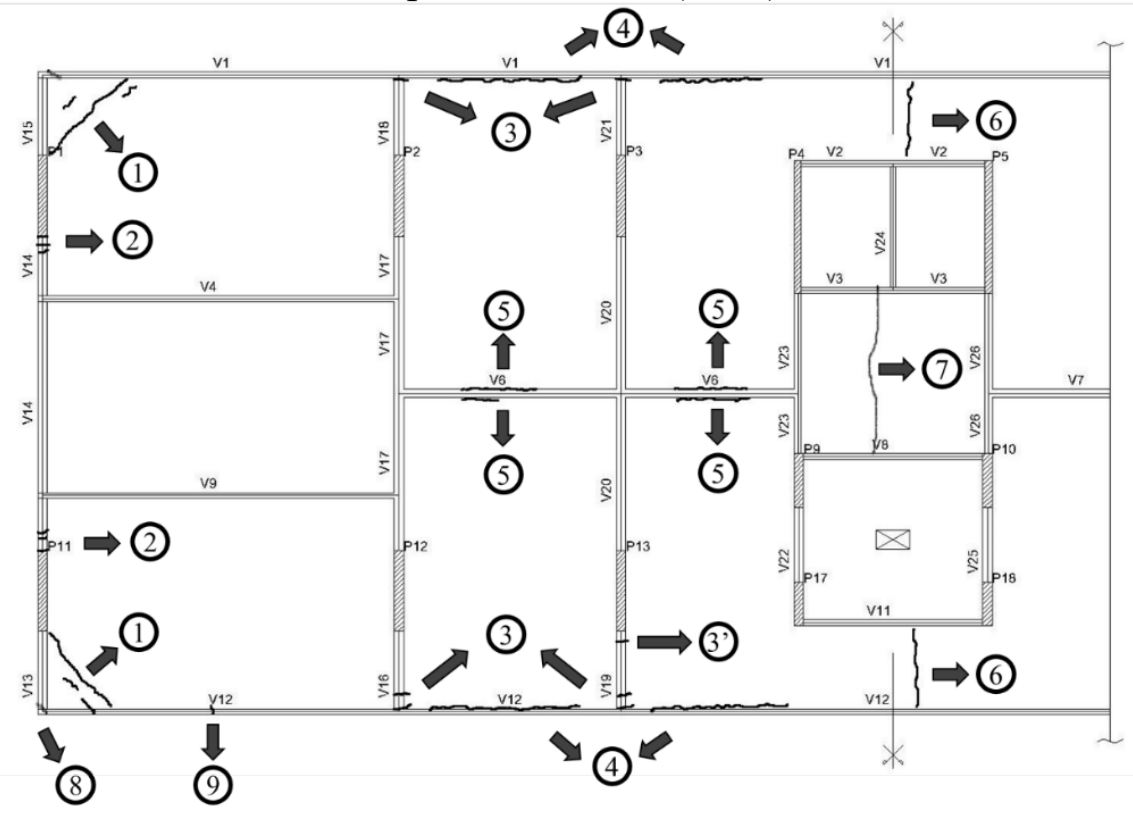

Figura 5. Dia 10: situação crítica. Fonte: adaptado de Fusco (1996).

No décimo dia de observação o panorama era extremamente crítico, as fissuras tipo 6 e 7 em um dos edifícios se prolongavam pelas alvenarias e se estendiam por quatro andares consecutivos. Além disso, havia evidências do seccionamento inteiro das vigas longitudinais da fachada (fissuras tipo 8 e 9). Com todas as observações relatadas, surgiu a hipótese de que os edifícios tendiam a se partirem em dois pedaços (Figura 5). Além disso, observou-se que a fissuração não tinha uma tendência clara de agravamento em função da posição do pavimento, o que dificultou na interpretação dos problemas encontrados. Então, uma intervenção provisória foi realizada como medida emergencial. Posteriormente foi realizado um processo de recuperação e reforço estrutural, que é relatado em Fusco (1996) e não será discutido no presente trabalho por não ser alvo do estudo. 


\section{MODELAGEM ESTRUTURAL}

A modelagem numérica da estrutura foi realizada utilizando o pacote computacional Eberick 2020 - demo (versão demonstrativa) e uma visualização tridimensional é apresentada na Figura 6. Ressalta-se que esta versão do programa utiliza as edições vigentes da NBR 6118 (2014) e NBR 6120 (2019) e que as assumpções a seguir são as mesmas do trabalho de Jesus et al. (2020).

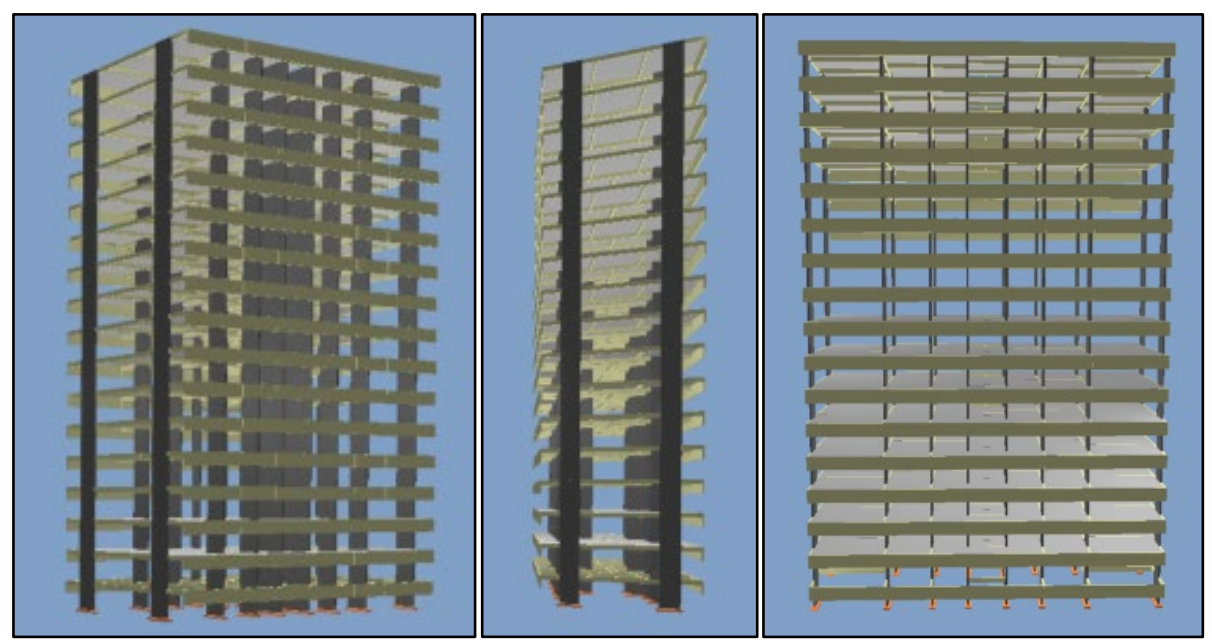

Figura 6. Modelo tridimensional da estrutura com uso do Eberick 2020 - demo.

Os edifícios têm características usualmente empregadas em centros urbanos. Assim, foi adotada classe de agressividade ambiental moderada e resistência característica à compressão do concreto $\left(f_{\mathrm{ck}}\right)$ de $25 \mathrm{MPa}$, sendo este o valor mínimo permitido pela NBR 6118 (2014). Conforme citado anteriormente, não há informações detalhadas do projeto arquitetônico, e por este motivo não é possível identificar o posicionamento dos cômodos. Assim, adotou-se uma carga acidental de $150 \mathrm{kgf} / \mathrm{m}^{2}$ e carga de revestimento de $150 \mathrm{kgf} / \mathrm{m}^{2}$, conforme prescrito pela NBR 6120 (2019). Considerou-se ainda que havia parede de alvenaria com $15 \mathrm{~cm}$ de espessura e peso específico de $1800 \mathrm{kgf} / \mathrm{m}^{3}$ sobre cada viga, também de acordo com a NBR 6120 (2019). Por fim, admitiu-se a fim de simplificar a concepção estrutural, que a escada seja considerada como uma laje.

Para calcular a carga devido à ação do vento utilizou-se os procedimentos indicados pela NBR 6123 (1988). Admitiu-se uma região de terreno plano ou pouco ondulado, ou seja, fator topográfico $\mathrm{S}_{1}=1,0$. A rugosidade do terreno foi admitida como categoria IV para o cálculo do fator geográfico $\mathrm{S}_{2}$, com nível do solo igual a $0,0 \mathrm{~m}$ e maior dimensão horizontal ou vertical entre $20 \mathrm{~m}$ e $50 \mathrm{~m}$. Além disso, admitiu-se que se tratava de uma edificação do tipo 2 para o fator estatístico (hotéis, residências, comércio ou indústria com alto fator de ocupação), sendo atribuído um valor de $\mathrm{S}_{3}=1,0$. Para definir a velocidade básica do vento, admitiu-se que os edifícios estavam localizados no estado de São Paulo, com vo $=40$ m/s de acordo com a NBR 6123 (1988).

Com isso, foi possível analisar a estabilidade estrutural dos edifícios por meio do parâmetro $\gamma_{z}$ (Tabela 1). Nesta análise é possível notar a estrutura é totalmente instável, apresentando um valor do parâmetro $\gamma_{\mathrm{z}}$ tendendo ao infinito para várias das combinações adotadas. Esta instabilidade é facilmente justificada, pois observando a planta de forma (Figura 1) é possível notar que não há boa formação de pórticos em nenhuma direção. É importante notar que Jesus et al. (2020) mostram que o coeficiente $\gamma_{z}$ tende a infinito mesmo quando a ação horizontal é muito baixa ( $\left.\mathrm{v}_{0}=1,0 \mathrm{~m} / \mathrm{s}\right)$. 
Tabela 1. Coeficiente $\gamma_{z}$ da estrutura.

\begin{tabular}{|c|c|c|c|c|c|c|}
\hline \multirow[t]{2}{*}{ Combinação } & \multicolumn{2}{|c|}{$\begin{array}{c}\text { Momento de } \\
\text { tombamento } \\
\text { de cálculo (tf.m) }\end{array}$} & \multicolumn{2}{|c|}{$\begin{array}{l}\text { Momento de } 2 \mathrm{a} \text {. } \\
\text { ordem } \\
\text { de cálculo (tf.m) }\end{array}$} & \multicolumn{2}{|c|}{ Gama-Z } \\
\hline & Eixo $X$ & Eixo Y & Eixo $X$ & Eixo Y & $\begin{array}{c}\text { Eixo } \\
\mathbf{X} \\
\end{array}$ & $\begin{array}{c}\text { Eixo } \\
\mathbf{Y}\end{array}$ \\
\hline $1,3 \mathrm{G} 1+1,4 \mathrm{G} 2+1,4 \mathrm{~S}+0,84 \mathrm{~V} 1+0,59 \mathrm{D} 1$ & 986,28 & 4086,08 & 2655,65 & 22,08 & $(*)$ & 1,01 \\
\hline $1,3 \mathrm{G} 1+1,4 \mathrm{G} 2+1,4 \mathrm{~S}+0,84 \mathrm{~V} 2+0,59 \mathrm{D} 2$ & 986,28 & 4086,08 & 2655,75 & 22,08 & $(*)$ & 1,01 \\
\hline $1,3 \mathrm{G} 1+1,4 \mathrm{G} 2+1,4 \mathrm{~S}+0,84 \mathrm{~V} 3+0,59 \mathrm{D} 3$ & 986,28 & 4086,08 & 0,03 & 647,23 & 1,00 & 1,19 \\
\hline $1,3 \mathrm{G} 1+1,4 \mathrm{G} 2+1,4 \mathrm{~S}+0,84 \mathrm{~V} 4+0,59 \mathrm{D} 4$ & 986,28 & 4086,08 & 0,03 & 647,24 & 1,00 & 1,19 \\
\hline $1,3 \mathrm{G} 1+1,4 \mathrm{G} 2+1,4 \mathrm{~S}+0,98 \mathrm{Q}+0,84 \mathrm{~V} 1+0,59 \mathrm{D} 1$ & 986,28 & 4086,08 & 2979,44 & 29,73 & $(*)$ & 1,01 \\
\hline $1,3 \mathrm{G} 1+1,4 \mathrm{G} 2+1,4 \mathrm{~S}+0,98 \mathrm{Q}+0,84 \mathrm{~V} 2+0,59 \mathrm{D} 2$ & 986,28 & 4086,08 & 2979,56 & 29,73 & $(*)$ & 1,01 \\
\hline $1,3 \mathrm{G} 1+1,4 \mathrm{G} 2+1,4 \mathrm{~S}+0,98 \mathrm{Q}+0,84 \mathrm{~V} 3+0,59 \mathrm{D} 3$ & 986,28 & 4086,08 & 0,04 & 731,16 & 1,00 & 1,22 \\
\hline $1,3 \mathrm{G} 1+1,4 \mathrm{G} 2+1,4 \mathrm{~S}+0,98 \mathrm{Q}+0,84 \mathrm{~V} 4+0,59 \mathrm{D} 4$ & 986,28 & 4086,08 & 0,04 & 731,17 & 1,00 & 1,22 \\
\hline $1,3 \mathrm{G} 1+1,4 \mathrm{G} 2+1,4 \mathrm{~S}+0,98 \mathrm{Q}+1,4 \mathrm{~V} 1+0,36 \mathrm{D} 1$ & 1643,80 & 6810,13 & 4965,71 & 29,73 & $(*)$ & 1,00 \\
\hline $1,3 \mathrm{G} 1+1,4 \mathrm{G} 2+1,4 \mathrm{~S}+0,98 \mathrm{Q}+1,4 \mathrm{~V} 2+0,36 \mathrm{D} 2$ & 1643,80 & 6810,13 & 4965,90 & 29,73 & $(*)$ & 1,00 \\
\hline $1,3 \mathrm{G} 1+1,4 \mathrm{G} 2+1,4 \mathrm{~S}+0,98 \mathrm{Q}+1,4 \mathrm{~V} 3+0,36 \mathrm{D} 3$ & 1643,80 & 6810,13 & 0,05 & 1200,60 & 1,00 & 1,21 \\
\hline $1,3 \mathrm{G} 1+1,4 \mathrm{G} 2+1,4 \mathrm{~S}+0,98 \mathrm{Q}+1,4 \mathrm{~V} 4+0,36 \mathrm{D} 4$ & 1643,80 & 6810,13 & 0,05 & 1198,79 & 1,00 & 1,21 \\
\hline $1,3 \mathrm{G} 1+1,4 \mathrm{G} 2+1,4 \mathrm{~S}+1,4 \mathrm{Q}+0,84 \mathrm{~V} 1+0,36 \mathrm{D} 1$ & 986,28 & 4086,08 & 3118,21 & 33,33 & $(*)$ & 1,01 \\
\hline $1,3 \mathrm{G} 1+1,4 \mathrm{G} 2+1,4 \mathrm{~S}+1,4 \mathrm{Q}+0,84 \mathrm{~V} 2+0,36 \mathrm{D} 2$ & 986,28 & 4086,08 & 3118,33 & 33,33 & $(*)$ & 1,01 \\
\hline $1,3 \mathrm{G} 1+1,4 \mathrm{G} 2+1,4 \mathrm{~S}+1,4 \mathrm{Q}+0,84 \mathrm{~V} 3+0,36 \mathrm{D} 3$ & 986,28 & 4086,08 & 0,05 & 767,46 & 1,00 & 1,23 \\
\hline $1,3 \mathrm{G} 1+1,4 \mathrm{G} 2+1,4 \mathrm{~S}+1,4 \mathrm{Q}+0,84 \mathrm{~V} 4+0,36 \mathrm{D} 4$ & 986,28 & 4086,08 & 0,05 & 767,47 & 1,00 & 1,23 \\
\hline $1,3 \mathrm{G} 1+1,4 \mathrm{G} 2+1,4 \mathrm{~S}+1,4 \mathrm{~V} 1+0,36 \mathrm{D} 1$ & 1643,80 & 6810,13 & 4426,06 & 22,08 & $(*)$ & 1,00 \\
\hline $1,3 \mathrm{G} 1+1,4 \mathrm{G} 2+1,4 \mathrm{~S}+1,4 \mathrm{~V} 2+0,36 \mathrm{D} 2$ & 1643,80 & & 4426,23 & 22,09 & $(*)$ & 1,00 \\
\hline $1,3 \mathrm{G} 1+1,4 \mathrm{G} 2+1,4 \mathrm{~S}+1,4 \mathrm{~V} 3+0,36 \mathrm{D} 3$ & 1643,80 & 6810,13 & 0,04 & 1067,83 & 1,00 & 1,19 \\
\hline $1,3 \mathrm{G} 1+1,4 \mathrm{G} 2+1,4 \mathrm{~S}+1,4 \mathrm{~V} 4+0,36 \mathrm{D} 4$ & 1643,80 & 6810,13 & 0,04 & 1064,00 & 1,00 & 1,19 \\
\hline $\mathrm{G} 1+\mathrm{G} 2+\mathrm{S}+0,84 \mathrm{~V} 1+0,59 \mathrm{D} 1$ & 986,28 & 4086,08 & 1969,45 & 12,17 & $(*)$ & 1,00 \\
\hline $\mathrm{G} 1+\mathrm{G} 2+\mathrm{S}+0,84 \mathrm{~V} 2+0,59 \mathrm{D} 2$ & 986,28 & 4086,08 & 1969,53 & 12,17 & $(*)$ & 1,00 \\
\hline $\mathrm{G} 1+\mathrm{G} 2+\mathrm{S}+0,84 \mathrm{~V} 3+0,59 \mathrm{D} 3$ & 986,28 & 4086,08 & 0,02 & 476,00 & 1,00 & 1,13 \\
\hline $\mathrm{G} 1+\mathrm{G} 2+\mathrm{S}+0,84 \mathrm{~V} 4+0,59 \mathrm{D} 4$ & 986,28 & 4086,08 & 0,02 & 475,80 & 1,00 & 1,13 \\
\hline $\mathrm{G} 1+\mathrm{G} 2+\mathrm{S}+0,98 \mathrm{Q}+0,84 \mathrm{~V} 1+0,59 \mathrm{D} 1$ & 986,28 & 4086,08 & 2293,24 & 17,99 & $(*)$ & 1,00 \\
\hline $\mathrm{G} 1+\mathrm{G} 2+\mathrm{S}+0,98 \mathrm{Q}+0,84 \mathrm{~V} 2+0,59 \mathrm{D} 2$ & 986,28 & 4086,08 & 2293,33 & 17,99 & $(*)$ & 1,00 \\
\hline $\mathrm{G} 1+\mathrm{G} 2+\mathrm{S}+0,98 \mathrm{Q}+0,84 \mathrm{~V} 3+0,59 \mathrm{D} 3$ & 986,28 & 4086,08 & 0,03 & 557,89 & 1,00 & 1,16 \\
\hline $\mathrm{G} 1+\mathrm{G} 2+\mathrm{S}+0,98 \mathrm{Q}+0,84 \mathrm{~V} 4+0,59 \mathrm{D} 4$ & 986,28 & 4086,08 & 0,03 & 557,90 & 1,00 & 1,16 \\
\hline $\mathrm{G} 1+\mathrm{G} 2+\mathrm{S}+0,98 \mathrm{Q}+1,4 \mathrm{~V} 1+0,36 \mathrm{D} 1$ & 1643,80 & 6810,13 & 3822,06 & 17,99 & $(*)$ & 1,00 \\
\hline $\mathrm{G} 1+\mathrm{G} 2+\mathrm{S}+0,98 \mathrm{Q}+1,4 \mathrm{~V} 2+0,36 \mathrm{D} 2$ & 1643,80 & 6810,13 & 3822,21 & 17,99 & $(*)$ & 1,00 \\
\hline $\mathrm{G} 1+\mathrm{G} 2+\mathrm{S}+0,98 \mathrm{Q}+1,4 \mathrm{~V} 3+0,36 \mathrm{D} 3$ & 1643,80 & 6810,13 & 0,03 & 924,49 & 1,00 & 1,16 \\
\hline $\mathrm{G} 1+\mathrm{G} 2+\mathrm{S}+0,98 \mathrm{Q}+1,4 \mathrm{~V} 4+0,36 \mathrm{D} 4$ & 1643,80 & 6810,13 & 0,03 & 917,84 & 1,00 & 1,16 \\
\hline $\mathrm{G} 1+\mathrm{G} 2+\mathrm{S}+1,4 \mathrm{Q}+0,84 \mathrm{~V} 1+0,36 \mathrm{D} 1$ & 986,28 & 4086,08 & 2432,01 & 20,81 & $(*)$ & 1,01 \\
\hline $\mathrm{G} 1+\mathrm{G} 2+\mathrm{S}+1,4 \mathrm{Q}+0,84 \mathrm{~V} 2+0,36 \mathrm{D} 2$ & 986,28 & 4086,08 & 2432,11 & 20,81 & $(*)$ & 1,01 \\
\hline $\mathrm{G} 1+\mathrm{G} 2+\mathrm{S}+1,4 \mathrm{Q}+0,84 \mathrm{~V} 3+0,36 \mathrm{D} 3$ & 986,28 & 4086,08 & 0,03 & 593,41 & 1,00 & 1,17 \\
\hline $\mathrm{G} 1+\mathrm{G} 2+\mathrm{S}+1,4 \mathrm{Q}+0,84 \mathrm{~V} 4+0,36 \mathrm{D} 4$ & 986,28 & 4086,08 & 0,03 & 593,41 & 1,00 & 1,17 \\
\hline $\mathrm{G} 1+\mathrm{G} 2+\mathrm{S}+1,4 \mathrm{~V} 1+0,36 \mathrm{D} 1$ & 1643,80 & 6810,13 & 3282,41 & 12,18 & $(*)$ & 1,00 \\
\hline $\mathrm{G} 1+\mathrm{G} 2+\mathrm{S}+1,4 \mathrm{~V} 2+0,36 \mathrm{D} 2$ & 1643,80 & 6810,13 & 3282,54 & 12,18 & $(*)$ & 1,00 \\
\hline $\mathrm{G} 1+\mathrm{G} 2+\mathrm{S}+1,4 \mathrm{~V} 3+0,36 \mathrm{D} 3$ & 1643,80 & 6810,13 & 0,02 & 794,73 & 1,00 & 1,13 \\
\hline $\mathrm{G} 1+\mathrm{G} 2+\mathrm{S}+1,4 \mathrm{~V} 4+0,36 \mathrm{D} 4$ & 1643,80 & 6810,13 & 0,02 & 784,88 & 1,00 & 1,13 \\
\hline
\end{tabular}


Siglas:

G1 - peso próprio;

G2 - carga permanente adicional;

$\mathrm{S}$ - empuxo do solo;

$\mathrm{Q}$ - carga acidental;

A - empuxo de água;

$\mathrm{V} 1$ - vento $\mathrm{X}+$;

$\mathrm{V} 2$ - vento $\mathrm{X}-$;

$\mathrm{V} 3$ - vento $\mathrm{Y}+$;

$\mathrm{V} 4$ - vento $\mathrm{Y}-$;

$(*)-\gamma_{\mathrm{z}}$ tende ao infinito (estrutura instável).

\section{RESULTADOS}

A partir da análise estrutural é possível justificar algumas das manifestações patológicas apresentadas pelos quatro edifícios. Além de a estrutura ser muito instável, conforme mostrado anteriormente pela análise de estabilidade global e no trabalho de Jesus et al. (2020), a distribuição dos elementos estruturais levou à distribuição inadequada dos esforços.

As fissuras tipo 1 (Figura 2) podem ser justificadas devido à ação do momento torçor presente nas vigas dessa região, conforme pode ser visto nas Figuras 7 e 8.

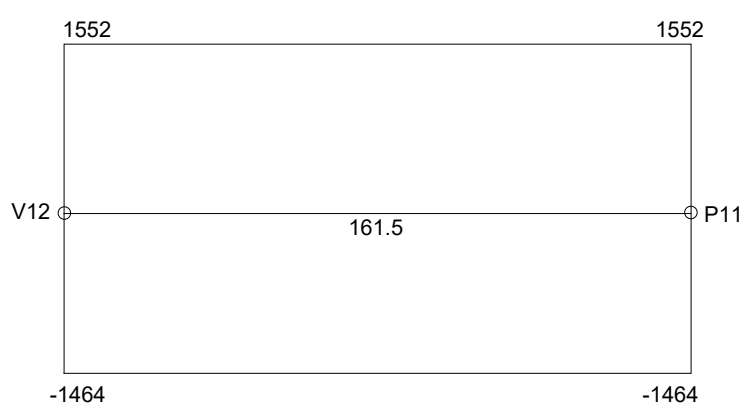

Figura 7. Envoltória de momentos torçores (kgf.m) na viga V13 via Eberick 2020 - demo.

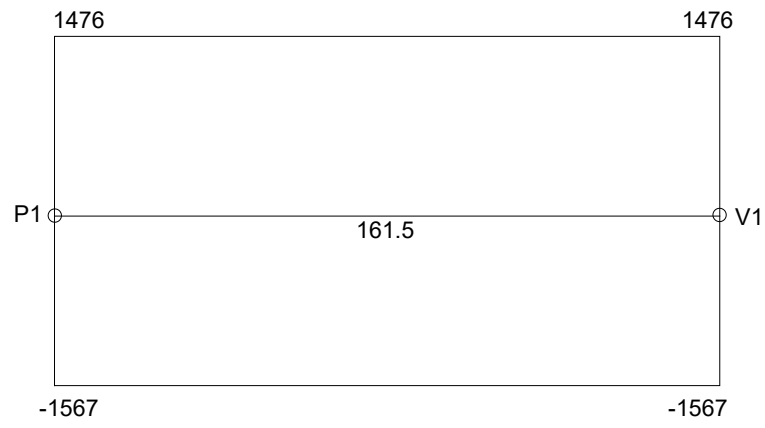

Figura 8. Envoltória de momentos torçores (kgf.m) na viga V15 via Eberick 2020 - demo.

Por sua vez, as fissuras do tipo 2, que ocorrem tendendo a separar as alvenarias e pilares, aparecem devido à má formação de pórticos que levou a parte da estrutura do pavimento a estar em balanço. 
Esta observação pode ser notada no diagrama de momentos fletores da viga V14 (Figura 9), no qual verifica-se a presença de alto valor de momento negativo na região dos pilares P1 e P11.

O mesmo problema anteriormente relatado, causado devido a concepção inadequada de pórticos e gerando distribuição inadequada dos esforços, pode justificar a aparição das fissuras tipo 3, 3', 4 e 5. Isto pode ser observado na Figura 10, que mostra o diagrama de momentos fletores na viga V19, onde nota-se novamente altos valores de momentos fletores na região próxima ao pilar P13.

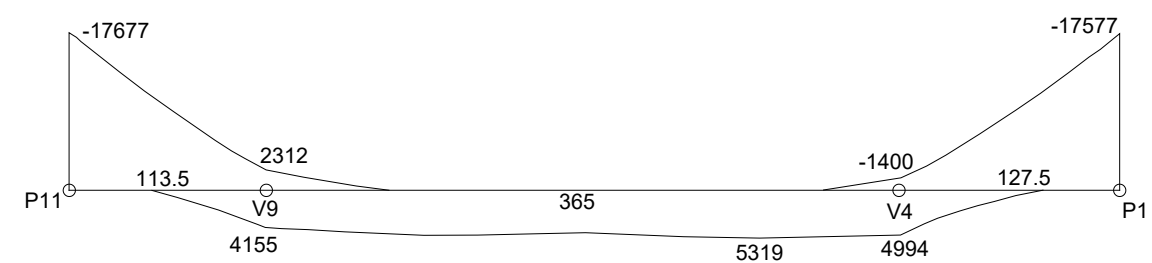

Figura 9. Envoltória de momentos fletores (kgf.m) na viga V14 via Eberick 2020 - demo.

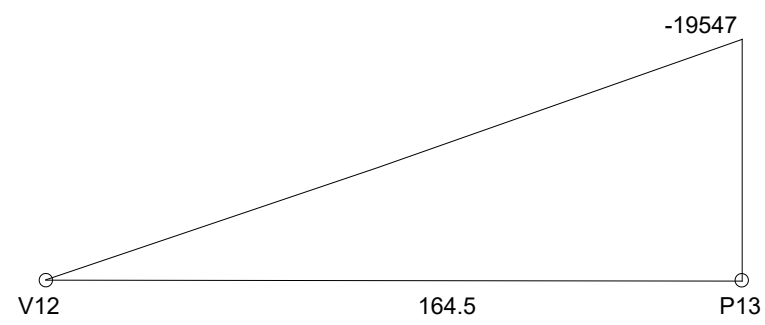

Figura 10. Envoltória de momentos fletores (kgf.m) na viga V19. Fonte: Eberick 2020 - demo.

Assim, observa-se que, em módulo, o momento fletor negativo de cálculo máximo na viga V14 é 17577,00 kgf.m. Devido a existência de sucessivos erros de concepção estrutural, o programa Eberick 2020 - demo não permite avaliar os estados limites de serviço, pois a condição de estabilidade da estrutura e alguns dos estados limites últimos de elementos estruturais específicos não foram atendidos. Desta forma, estimou-se que o momento fletor negativo atuante para a combinação rara é 12626,43 kgf.m, que é uma ordem de grandeza maior do que o momento de fissuração (resistente) para esta viga, cujo cálculo resultou em 1318,23 kgf.m.

Ainda devido à má formação de pórticos que implica em parte do pavimento estar em balanço, é possível notar uma tendência de levantamento das vigas V6 e V7. Esta constatação é confirmada observando-se o carregamento de ambas as vigas, que mostra a presença carregamento negativo, levando assim a manifestação das fissuras do tipo 5.

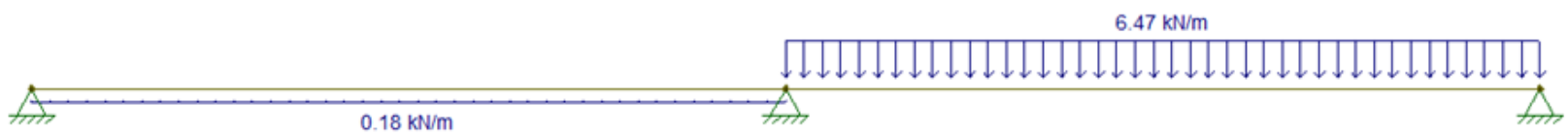

Figura 11. Carregamento nas vigas V6 e V7 obtidas com Eberick 2020 - demo. Esquema estrutural via Ftool.

A viga em análise (V6) tem $16 \mathrm{~cm}$ de largura e $50 \mathrm{~cm}$ de altura. Admitindo que esta foi construída com concreto de propriedades similares às demais analisadas, tem-se $f_{c k}=25 \mathrm{MPa}$. Com isso, é obtida a seguinte configuração deformada, com máximo deslocamento negativo (para cima) aproximadamente $0,2 \mathrm{~mm}$. 


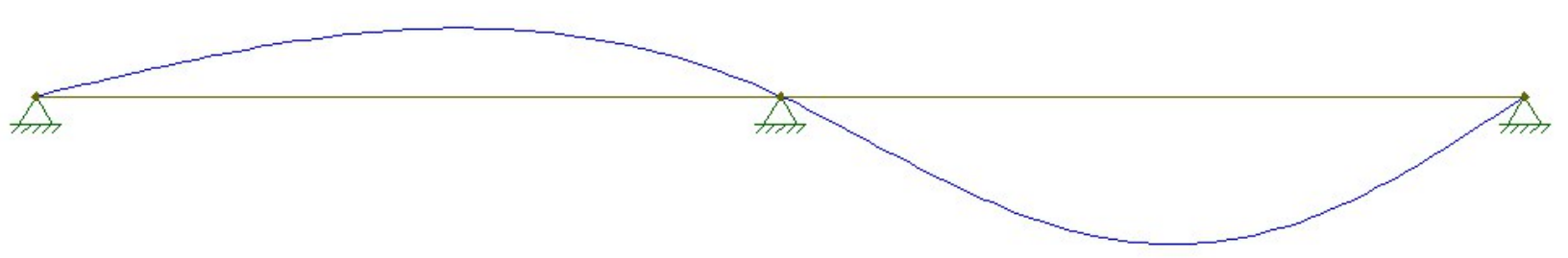

Figura 12. Aspecto deformado da viga V6 via Ftool.

Visto que surge uma fissura horizontal abaixo da viga V6 (Figura 13), exatamente na ligação desta com a alvenaria, analisa-se se a tensão atuante nesta alvenaria é maior que a tensão de aderência da mesma. Considerando que a fissura se deu na alvenaria, são assumidos 0,28MPa e 8,05GPa para resistência à aderência e módulo de elasticidade, respectivamente (Silva; Campiteli, 2008). Assim, a maior tensão atuante na ligação entre a alvenaria e a viga V6 é $67,98 \mathrm{MPa}$, valor muito maior do que a resistência admitida.

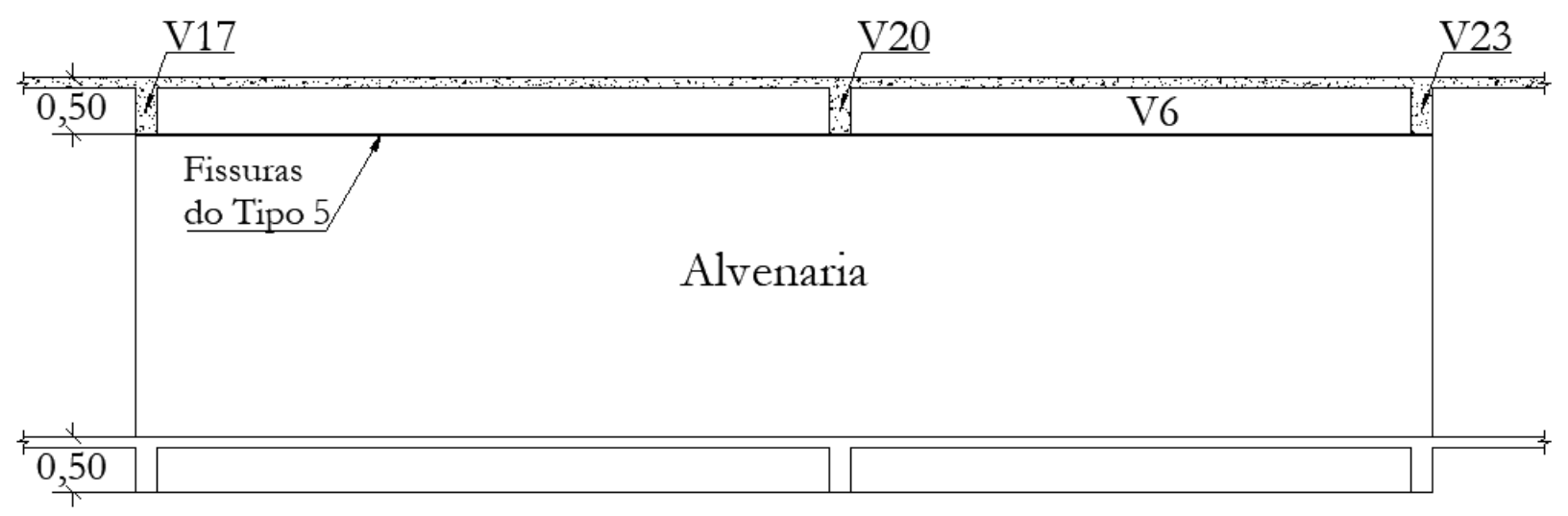

Figura 13. Fissuras tipo 5, conforme Fusco (1996).

As fissuras do tipo 6 e 7 são indicações diretas da alta instabilidade estrutural dos edifícios, constatada na análise de estabilidade global. Conforme relatado em Fusco (1996), estas fissuras nas observações finais se estendiam por vários pavimentos e tendiam a separar as edificações em duas partes. Por fim, as fissuras 8 e 9 são decorrentes dos efeitos secundários da evolução de todas as fissuras anteriormente relatadas.

\section{CONCLUSÕES}

Neste trabalho apresentou-se um estudo de caso no qual se analisou as manifestações patológicas causadas devido à problemas com estabilidade global e má concepção estrutural de um edifício alto. As fissuras relatadas apresentaram rápida evolução em um curto espaço de tempo após a estrutura entrar em serviço.

Os resultados apresentados mostraram que os efeitos de segunda ordem eram extremamente altos, levando o coeficiente $\gamma_{\mathrm{z}}$ tender ao infinito em várias das combinações de ações adotadas.

Ao analisar o comportamento mecânico da estrutura foi possível notar a inadequada distribuição de esforços por todo o pavimento. As manifestações de algumas fissuras foram consequência da presença de momento volvente na laje, que foi mostrada no presente trabalho pela presença de momentos torçores nas vigas. Outras fissuras foram justificadas devido à grande parte da estrutura 
estar em balanço, o que levou a geração de altos momentos negativos em algumas vigas e carregamento negativo nas vigas centrais.

Por fim, diante do exposto no presente trabalho, é possível notar que a má concepção estrutural levou a manifestações patológicas severas no edifício analisado. Neste caso, a falha catastrófica da estrutura foi evitada por meio de recuperação estrutural.

\section{AGRADECIMENTOS}

O terceiro e o quinto autores agradecem ao Programa de Pós-Graduação em Engenharia Civil da Universidade Federal de Alagoas (PPGEC/UFAL) pelo aporte. O primeiro autor agradece a Coordenação de Aperfeiçoamento de Pessoal de Nível Superior (CAPES) pelo suporte financeiro durante seu curso de mestrado. O terceiro autor agradece a CAPES pelo suporte financeiro durante seu curso de doutorado. A quarta autora agradece a PNPD/CAPES pelo suporte financeiro durante seu curso de pós-doutorado.

\section{REFERENCIAS}

Associação Brasileira de Normas Técnicas (ABNT). (1988). NBR 6123: Forças devidas ao vento em edificações. Rio de Janeiro.

Associação Brasileira de Normas Técnicas (ABNT). (2014). NBR 6118: Projeto de estruturas de concreto - Procedimento. Rio de Janeiro.

Associação Brasileira de Normas Técnicas (ABNT). (2019). NBR 6120: Cargas para o cálculo de estruturas de edificações. Rio de Janeiro.

Beck, H., König, G. (1966), "Restraining forces in the analysis of tall buildings" in: Symposium On Tall Buildings, Pergamon Press, Oxford, p. 513-536.

Bueno, J. R., Loriggio, D. D. (2016) Análise de efeitos de $2^{\mathrm{a}}$ ordem: estudo de caso. Revista IBRACON de Estruturas e Materiais. 9(4):494-509. https://doi.org/10.1590/S198341952016000400002.

Campoó, L. B., Corrêa, M. R. S., Ramalho, M. A. (2005), Efeitos de segunda ordem em edifícios de alvenaria estrutural. Revista Minerva. 2(2):173-184.

Carmo, R. M. S., Corrêa, M. R. S.; Ramalho, M. A. (1995), Correlação entre parâmetros de estabilidade global de estruturas de edifícios in: Jornadas Sudamericanas de Ingenieria Estructural, Associação Sul-Americana de Engenharia Estrutural, Tucuman: pp. 99-110.

Cunha, R. N., Mendes, L. A., Amorim, D. L. N. F. (2020), Proposta de critério simplificado para estimar efeitos de segunda ordem em edifícios de concreto armado. Revista IBRACON de Estruturas e Materiais. 13(2):200-211. https://doi.org/10.1590/S1983-41952020000200002.

Jesus, L. S., Teles, D. V. C., Santos, D. M., Amorim, D. L. N. F. (2020), Influência dos efeitos globais de segunda ordem em manifestações patológicas em um edifício de concreto, Revista Principia (João Pessoa). 51:97-111. http://dx.doi.org/10.18265/1517-0306a2020v1n51p97-111.

EBERICK 2020 - demo. Versão 13.285: AltoQi, 2020. Disponível em: https://www.altoqi.com.br/downloads/. Acesso em: 28 fev. 2020.

Ellwanger, R. J. (2019), Um limite variável para o parâmetro de instabilidade de estruturas de contraventamento formadas por associações de pórticos com paredes ou núcleos. Revista IBRACON de Estruturas e Materiais. 5(1):104-136. https://doi.org/10.1590/S198341952012000100008. 
Feitosa, L. A., Alves, E. C. (2015), Estudo da estabilidade global de edifícios altos com lajes protendidas. Revista IBRACON de Estruturas e Materiais. 8(2):196-224. https://doi.org/10.1590/S1983-41952015000200008.

Franco, M., Vasconcelos, A. C. (1991), Practical assessment of second order effects in tall buildings in: Colloquium on the CEB-FIP MC90, Rio de Janeiro, p. 307-324.

Freitas, F. C., Luchi, L. A. R., Ferreira, W. G. (2016), Análise da estabilidade global das estruturas e ações de controle dos seus efeitos. Revista IBRACON de Estruturas e Materiais. 9(2): 192-213. https://doi.org/10.1590/S1983-41952016000200003.

Ftool - Two-Dimensional Frame Analysis Tool. Versão 4.00.04: 2018. Disponível em: https://www.ftool.com.br/Ftool/user/download. Acesso em: 28 de maio de 2021.

Fusco, P. B. (1996), Patologia da Concepção Estrutural de Edifícios Altos. In: Cunha, A.J.P.; Lima, N.A.; Souza, V. C. M. “Acidentes estruturais na construção civil”. PINI, São Paulo, p. 212.

Menezes, J. P. R., Vieira, C. S., Amorim, D. L. N. F. (2020), Relação entre os coeficientes $\alpha$ e $\gamma z$ para edifícios altos de concreto armado e alvenaria estrutural. Revista Principia (João Pessoa). 51:158-165. http://dx.doi.org/10.18265/1517-0306a2020v1n51p158-165.

Silva, N. G., Campiteli, V. C. (2008), Módulo de elasticidade dinâmico de argamassa de revestimento in: II Encontro de Engenharia e Tecnologia dos Campos Gerais, Campos Gerais: pp. $1-9$.

Vieira, V. V. S; Rodrigues Jr, S. J.; Veloso, L. A. C. M. (2017), Análise da estabilidade global de edifícios de concreto armado utilizando o coeficiente $\gamma_{\mathrm{z}}$. Revista IBRACON de Estruturas $e$ Materiais. 10(5):1113-1140. https://doi.org/10.1590/S1983-41952017000500010. 